\section{Internet-Based Administration of Shared Instruments with Facility Online Manager}

\section{Shu-You Li and Vinayak P. Dravid}

Northwestern University, Evanston, IL

syli@northwestern.edu,v-dravid@northwestern.edu

Resource sharing has become an absolute necessity for modern scientific research because of the increasing expense and complexity of instruments, and the ever changing funding paradigm which often requires sharing of major instrumentation resource across multiple disciplines. Many universities and even large companies have set up centralized facilities to serve researchers from different departments, divisions and units to minimize expenses and maximizing usage.

Just in the materials research area alone, it is estimated that there are more than 500 mid-size facilities existing nationwide, as reported in 2005 by the Committee on Smaller Facilities of National Research Council [1]. Resource sharing is also common in large research groups, between Principal Investigators, as well as in corporate/industrial laboratories.
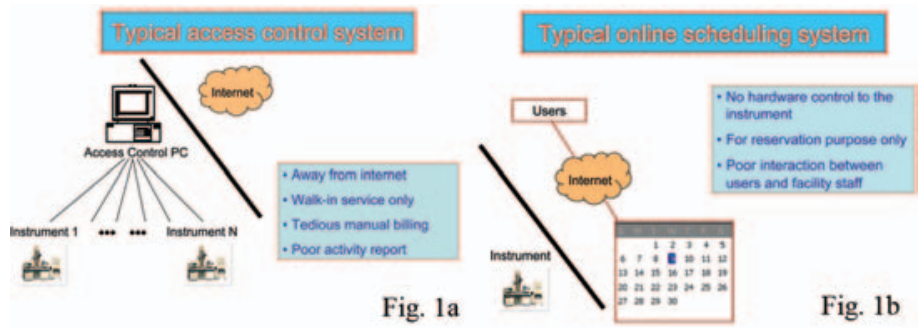

Fig. 1a. A typical access control system in a shared facility.

Fig. 1b. A typical online reservation system in a shared facility.

The universal need to maintain, manage, and share a diverse user base across multiple disciplines has made the difficult problem of managing instrumentation user facilities even more challenging. While enjoying the benefits of resource sharing, the managers of these facilities realize that they face a number of major challenges, including:

- How to control access to the facility? Daytime versus all-day?

- How to schedule the instruments efficiently?

- How to keep usage and maintenance records of the instruments?

- How to share or backup experimental data while keeping research secrets and privacy matters?

- How to inform technical staff when an instrument needs attention?

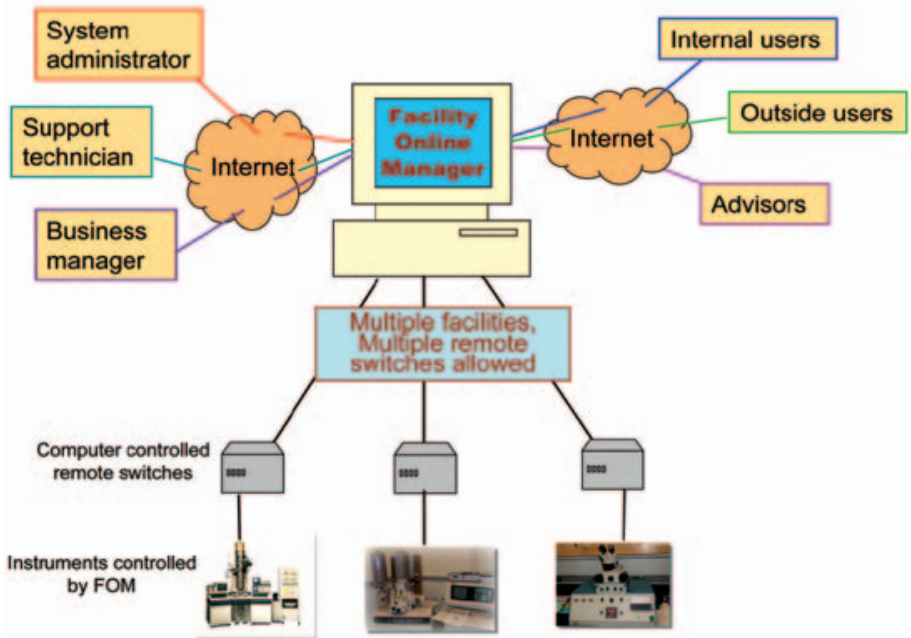

Fig. 2. The new FOM system designed and implemented in NUANCE
- How to maintain an up-to-date user database?

- How to get performance reports of the instruments' usage?

- How to manage allocation of costs associated with the instruments or invoice?

Further, with increasing federal scrutiny of grants and appropriate use of grant resources (i.e., federal compliance), there is an added pressure on facility directors and managers to ensure proper record upkeep and usage charge structures consistent with federal mandates.

There are mainly three categories of methods that facility managers are using to address these challenges:

1. Paper-based reservation and log sheets.

2. Local computer software to control instrument access (as shown in fig. 1a).

3. Online scheduling software to reserve instrument access (as shown in fig. $1 b$ ).

Only recently have the facility managers started considering complete accounting solutions with online applications. However, due to the lack of communications between microscopists and software developers, these online applications often result in less flexibility (dedicated for certain charge rate structure, certain facility use, for example) or less functionality (such as various financial reports, reservation limits, for instance).

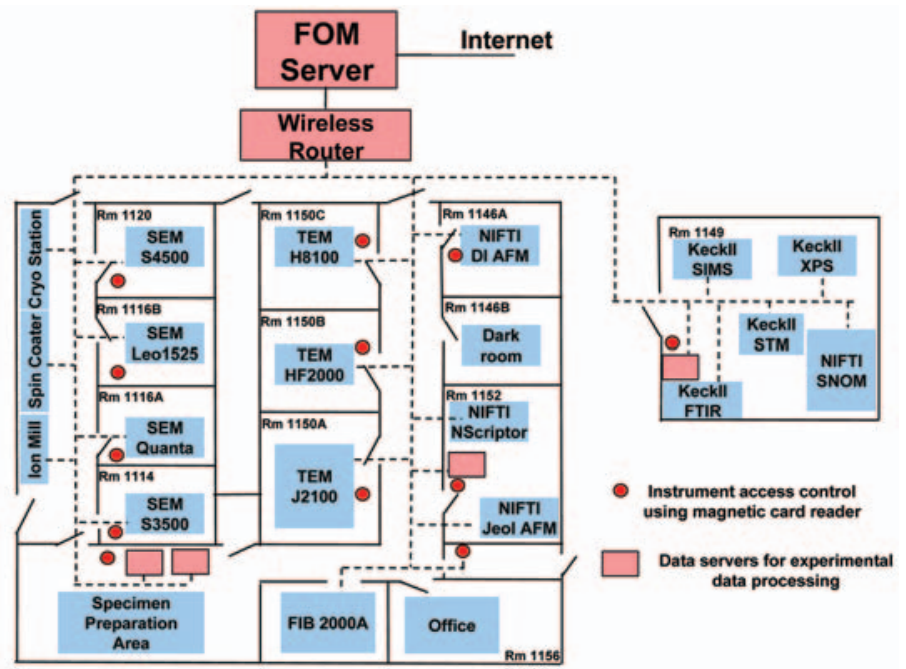

Fig. 3. FOM implementation at NUANCE, Northwestern University.

Northwestern University Atomic and Nanoscale Characterization Experimental Center (NUANCE) is the largest shared resource at Northwestern University, serving more than 500 users. It comprises three complementary facilities:

(i) EPIC (Electron Probe Instrumentation Center): Which houses several scanning, transmission and scanning-transmission electron microscopes, with a multitude of analytical and peripheral accessories, as well as a complete specimen preparation suite, including a focused ion beam (FIB) system.

(ii) Keck-II: A surface science facility supported by the Keck foundation that includes UHV surface science capabilities such as XPS, SIMS and STM/AFM.

(iii) NIFTI (Nanoscale Integrated Fabrication, Testing and Instrumentation) Facility: This principally covers lithography and scanning probe capabilities with several AFMs/STMs, e-beam lithography, and near-field scanning optical microscopy (NSOM) instrumentation.

Given the diversity of the three core facilities in NUANCE, the large user base, and an ever-increasing need to ensure proper data 


\section{Let 4pi take you beyond the capability of any SEM/STEM on the market with}

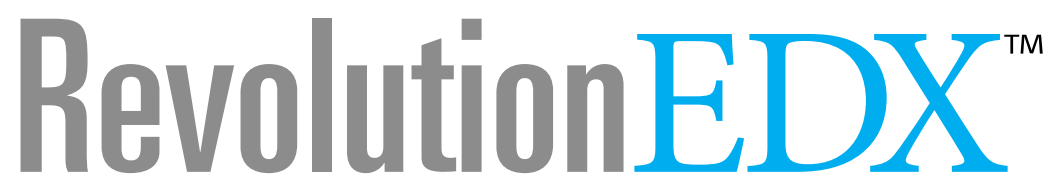

\section{X-ray Microanalysis Systems}

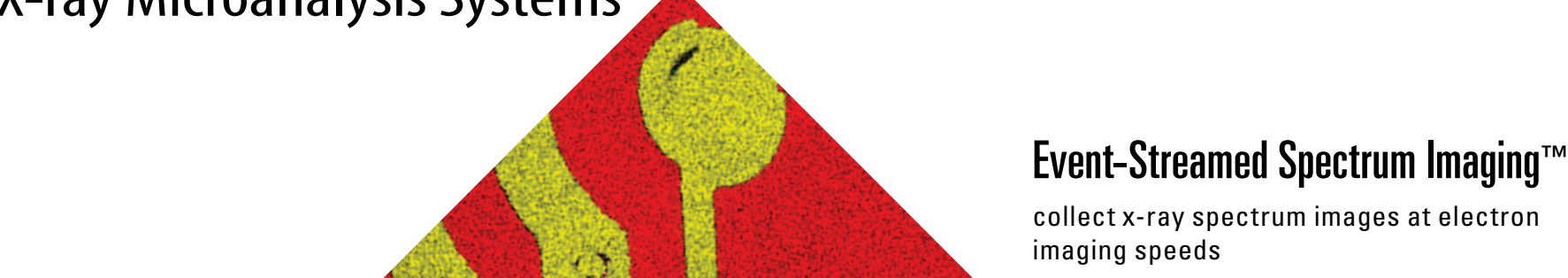

- Easy to use, one-click acquisition for spectra, images and maps

- High-resolution high-throughput Silicon-drift technology detectors-Nitrogen-free operation with a system that knows how to leverage the advantage

- Dynamic Dwell Modulation ${ }^{\mathrm{TM}}$

spend maximum time on areas of interest while continuing to obtain x-ray data from the entire area

- Robust auto peak-ID based on real-time full deconvolution of acquired x-ray spectra

Join the revolution in cutting-edge EDX technology

Available TODAY only from

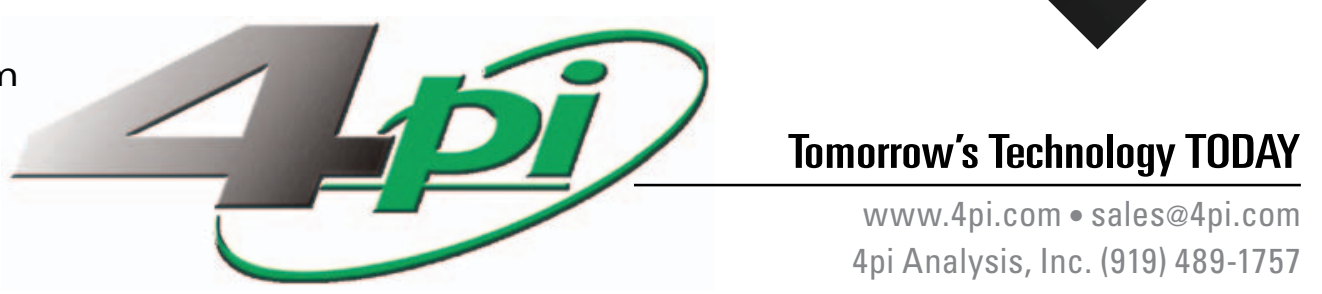



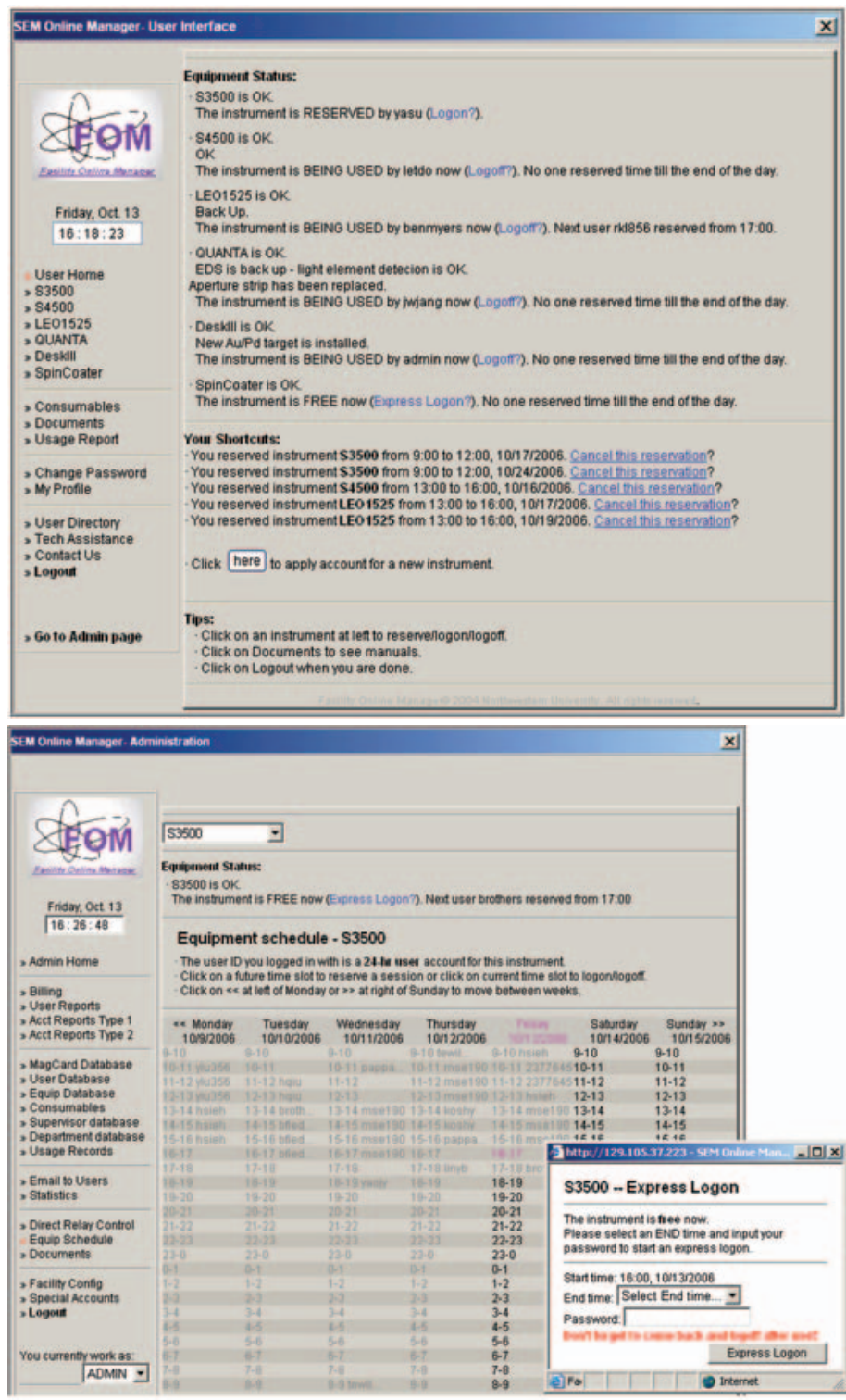

\section{सfक्ष}

\section{\&}

Friar, oct 13

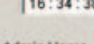

admin Home

;User Reports

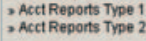

MagCard Database

User Database

Equip Database

> Consumables
3 Supenisor database
, Department database

s Usage Records

- Email to Users

- Direct Retar Control
Equip Schedule

: Equip Schedulo
, Documents

$\rightarrow$ Facility Confifg

S Special Accoumts
Logotif

You curenty work as:

Swatch between facilities
TEM

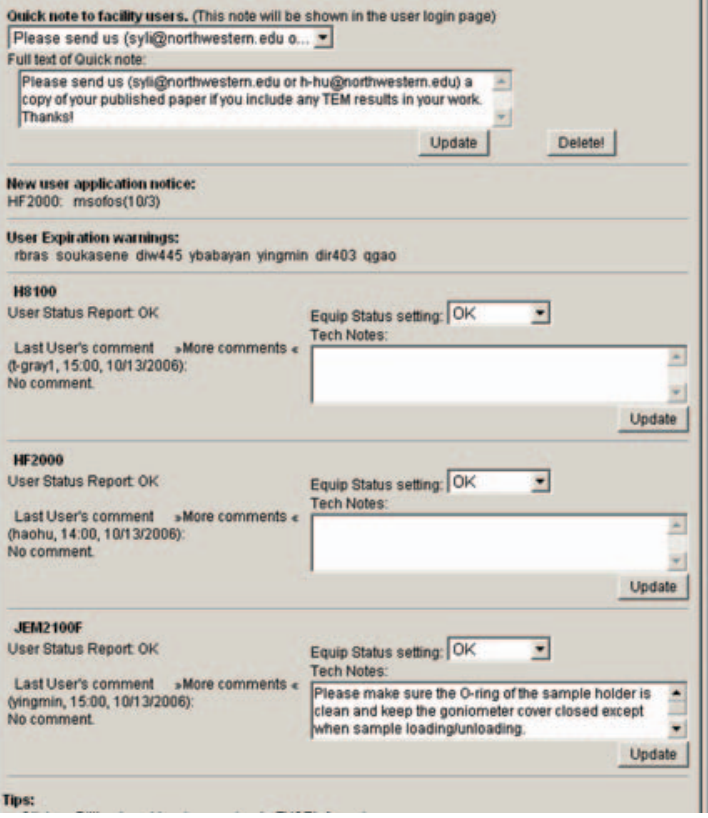

Fig. 4. Typical user interfaces of FOM when the user logs in (User Home, 4a), open instrument schedule (4b), and administrator log in (Admin Home). collection for several research centers at Northwestern, it became clear very quickly that we had to develop an "integrated solution" to facility operation and management, rather than rely on merely patch-work.

We have fully implemented an integrated and in-house developed software program, called Facility Online Manager (FOM $\left.{ }^{*}\right)$, to manage practically all aspects of NUANCE management and operation. FOM was developed and launched in 2003, by the authors who have their heart in microscopy, and thus are in complete tune with the needs, wishes and whims of all users, managers, sponsors and the executive administrators of these facilities. With three years of experience, we believe that the FOM ${ }^{\circ}$ software is quite mature and amenable to suit diverse needs in disparate facilities.

The major features of FOM now include:

- Easy-to-use user interface with customizable facility logo and other features

- Local area network for access control switches and data servers
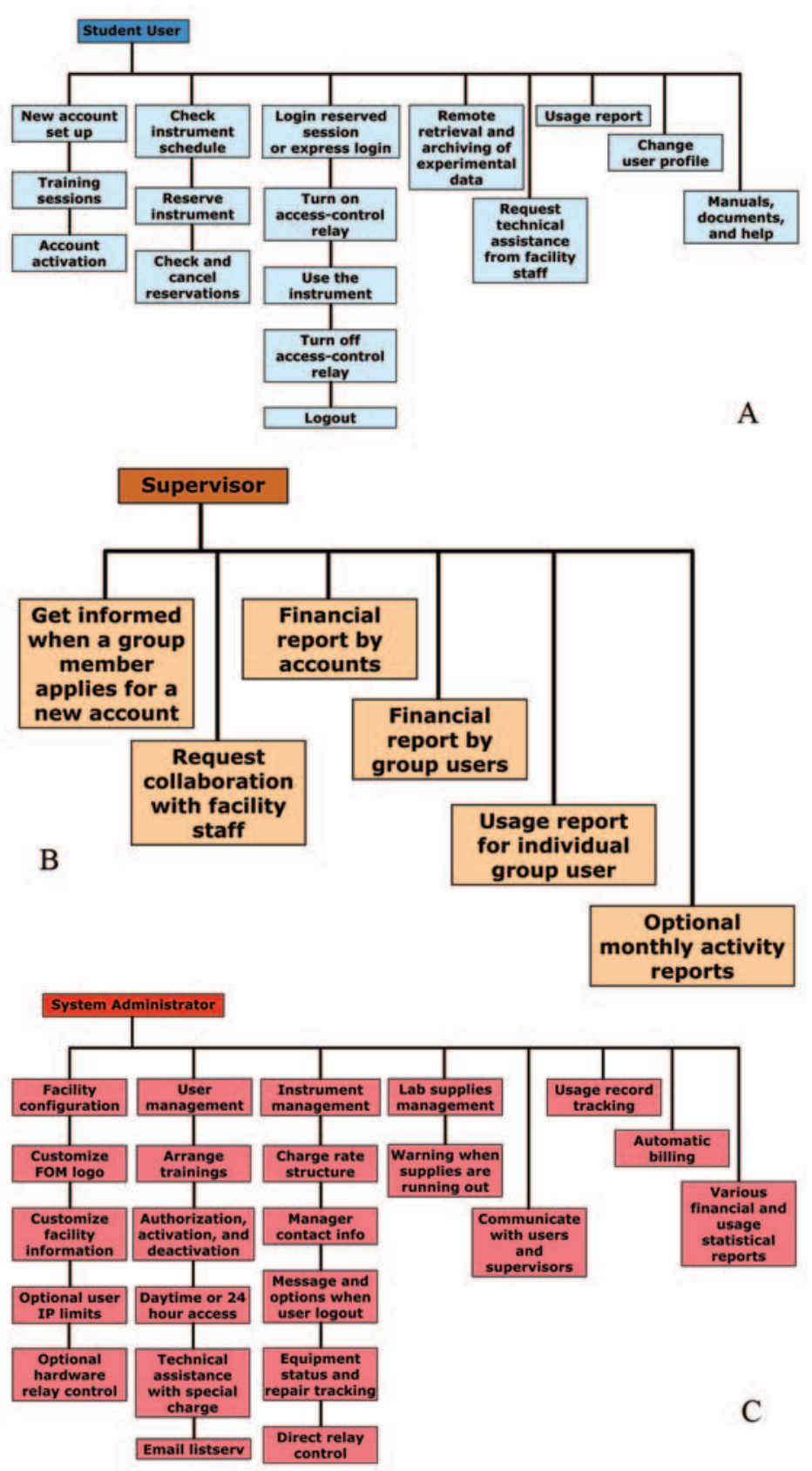

Fig. 5. Use case diagrams of FOM for student users (a), supervisors (b), and administrators of $\mathrm{FOM}^{\circ}(\mathrm{c})$. 
- Multiple user levels (daytime access or $24 \mathrm{hr}$ access) and customizable charge rates

- Magnetic card scanners for instruments access control

- Accounting and inventory records

- Password protected remote access to user's experimental data

- Performance reports of instrument usage

- Automated backup of management and user data.

- E-mail alerts for users, advisors and business managers

A schematic map of FOM ${ }^{\circ}$ as implementation at NUANCE is shown in fig. 3. We have installed magnetic card readers in every room. Users may reserve sessions with any internet-connected computer, just like common scheduling softwares do. However unlike other scheduling softwares, we allow log-on and use of the instruments with only authorized magnetic cards. This ultimately eliminated unauthorized access to the instruments.

Figure 4 shows a typical user interface. One can see links to authorized instruments for the current user and other functions in the left side of the window. The operation is fairly straightforward-users simply click on available time slots to reserve a session, and swipe the magnetic card just before use of the instrument.

As a reference for any facility that plans to design software themselves, we present our use-case diagrams for the FOM ${ }^{\circ}$ system in fig. 5. We have implemented all the use cases listed in FOM ${ }^{\circ}$. Additional use-case scenarios may be easily incorporated.

A free demo of the software is available at http://www.fom. northwestern.edu/.

\section{References}

[1] Midsize Facilities: Infrastructure for Materials Research, The National Academies Press, 2005.

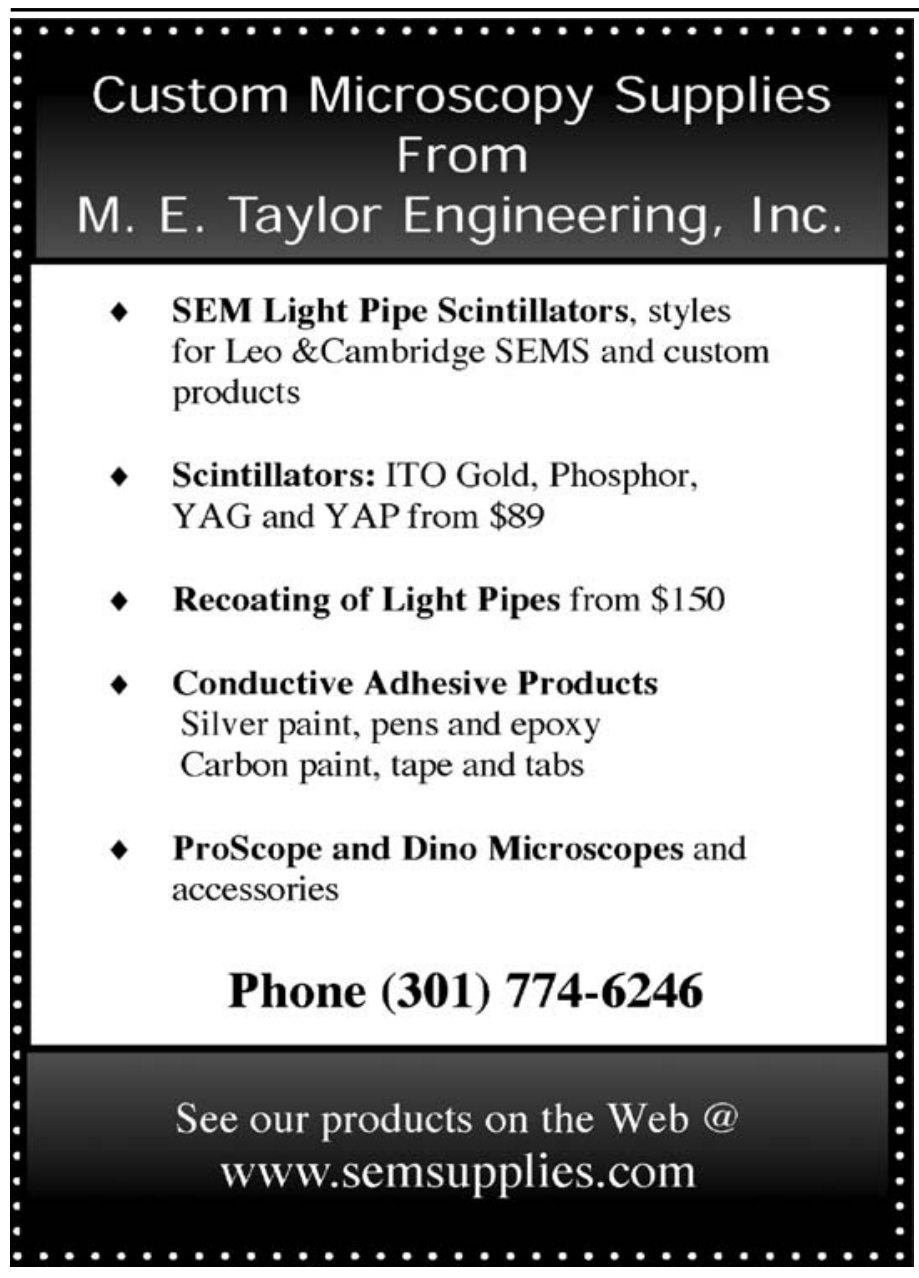

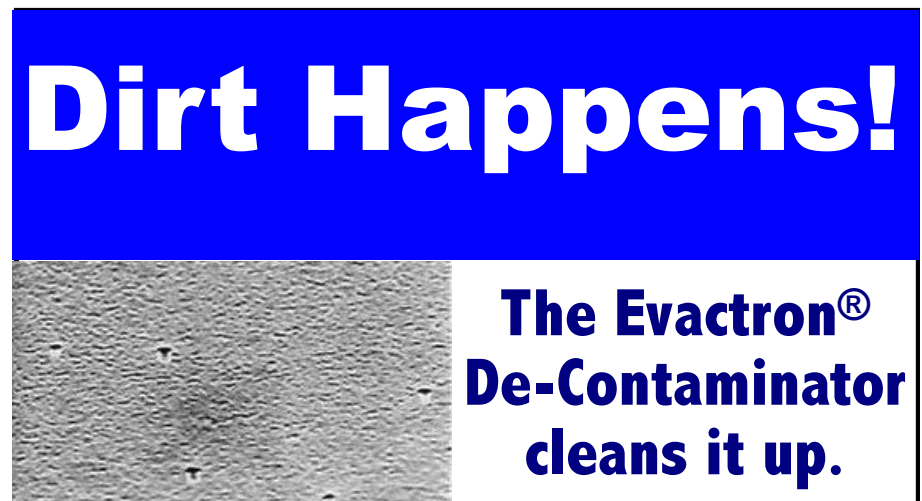

- After first Evactron cleaning for 10 minutes

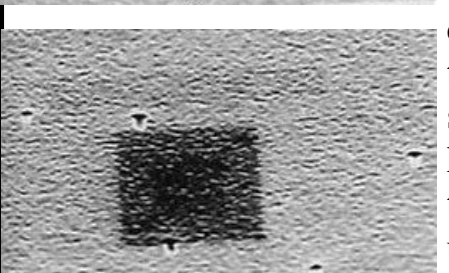

From the most persistent contamination problems to the subtlest, the Evactron system quickly removes the hydrocarbon dirt from Electron Microscopes and vacuum chambers no matter

AMD XL40 Before cleaning what the source.

The needs of nanotechnology have steadily pushed up the performance of FE SEMS in the last decade. We can see smaller details, but ever smaller amounts of dirt can interfere. Dry vacuum systems have become the standard of the industry, but hydrocarbon contamination problems persist because the world we live in provides a constant organic background. Like regular dusting and vacuuming cleans your home, regular use of the Evactron De-Contaminator will remove this dirt and keep it from becoming a problem.

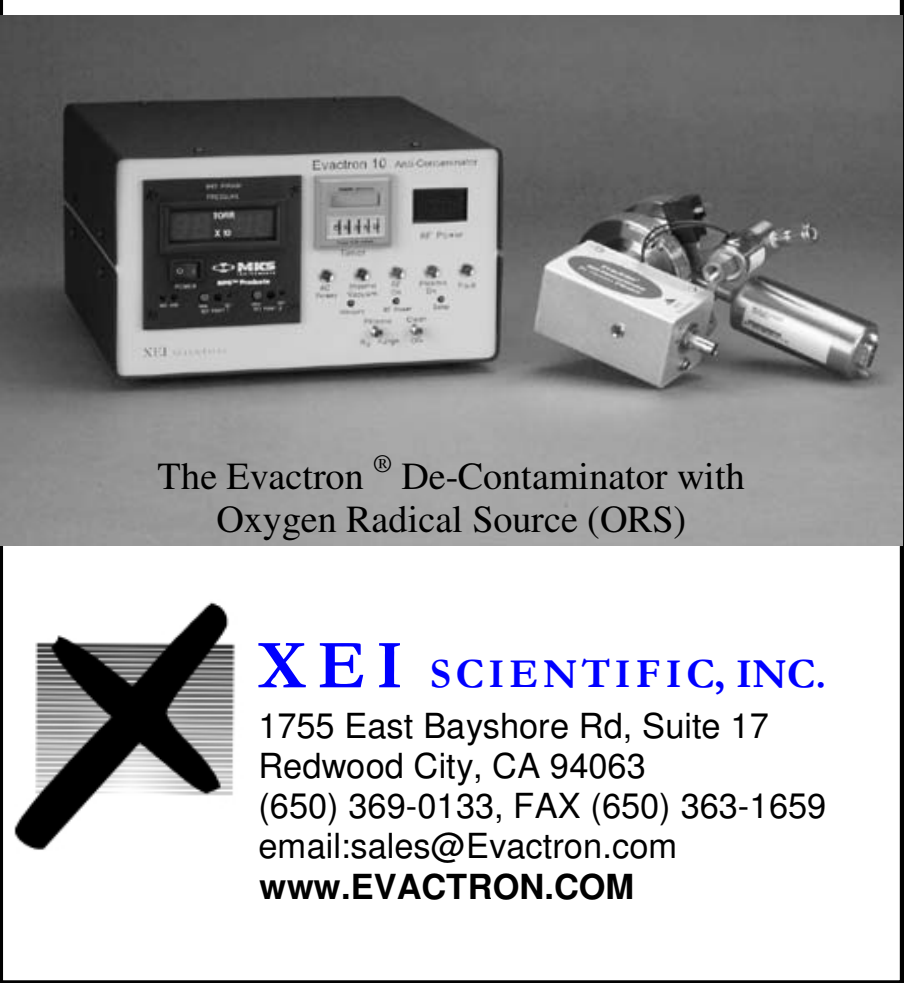

